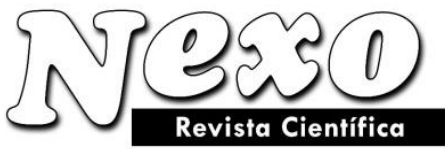

ISSN-E 1995-9516

Universidad Nacional de Ingeniería COPYRIGHT @ (UNI). TODOS LOS DERECHOS RESERVADOS

http://revistas.uni.edu.ni/index.php/Nexo https://doi.org/10.5377/nexo.v33i02.10799

Vol. 33, No. 02, pp. 638-650X/Diciembre 2020

(c) (i) $(-)$

\title{
Simulation of quadcopter flight altitude stabilization system
}

\section{Simulación del sistema de estabilización de altitud de vuelo en quadcopter}

\author{
Mikhail Yu. Babich ${ }^{1}$, Mikhail M. Butaev ${ }^{1}$, Dmitry V. Pashchenko ${ }^{2}$, Alexey I. Martyshkin ${ }^{3}$, \\ Dmitry A. Trokoz
}

${ }^{1}$ Dr. of Technical Sciences, Associate Professor, Department Main Specialist JSC Research and Production Enterprise «Rubin», (440000, Russia, Penza, Baydukova St, 2,

${ }^{2}$ Dr. of Technical Sciences, Professor, Rector, Penza State Technological University

(440039, Russia, Penza, 1/11 Baydukova proyezd/Gagarina ul., 1/11

${ }^{3}$ Candidate of Technical Sciences, Associate Professor, Department of Computational Machines and Systems, Penza State Technological University, 440039, Russia, Penza, 1/11 Baydukova proyezd/Gagarina ul., 1/11.

${ }^{4}$ Candidate of Technical Sciences, Associate Professor, Vice-Rector for Research, Penza State Technological University, (440039, Russia, Penza, 1/11 Baydukova proyezd/Gagarina ul., 1/11.

Corresponding author email: babichmj@mail.ru

(recibido/received: 16-July-2020; aceptado/accepted: 19-September-2020)

\begin{abstract}
Recently, unmanned aerial vehicles have been an important part of scientific research in various fields. Quadrocopter is an unmanned aerial vehicle with four rotors, two of which rotate clockwise, the other two counterclockwise. Changing the speed of screw rotation allows you to control the movement of the apparatus. The article proposed and tested a mathematical model of a quadcopter. They presented the development of a simple control algorithm that allows to stabilize the height and angular position. The research results show the efficiency of the algorithm and the possibility of its practical implementation. The developed mathematical model can be used instead of a real quadcopter, which will significantly reduce the time during research, as well as avoid the quadrocopter damage, reducing the number of launches.
\end{abstract}

Key words: quadrocopter, flight altitude stabilization, unmanned aerial vehicle, modeling, multicopter, algorithm, implementation.

\section{RESUMEN}

Recientemente, los vehículos aéreos no tripulados han sido una parte importante de la investigación científica en varios campos. Quadrocopter es un vehículo aéreo no tripulado con cuatro rotores, dos de los cuales giran en sentido horario y los otros dos en sentido antihorario. Cambiar la velocidad de rotación del tornillo le permite controlar el movimiento del aparato. El artículo propuso y probó un modelo matemático de un quadcopter. Presentaron el desarrollo de un algoritmo de control simple que permite estabilizar la altura y la posición angular. Los resultados de la investigación muestran la eficiencia del algoritmo y la 
posibilidad de su implementación práctica. El modelo matemático desarrollado se puede utilizar en lugar de un cuadricóptero real, lo que reducirá significativamente el tiempo durante la investigación, además de evitar el daño del cuadricóptero, reduciendo el número de lanzamientos.

Palabras clave: cuadricóptero, estabilización de altitud de vuelo, vehículo aéreo no tripulado, modelización, multicóptero, algoritmo, implementación.

\section{INTRODUCTION}

Recently, unmanned aerial vehicles (UAVs) have formed an important part of scientific research in various fields. UAVs are finding applications in various areas ranging from military applications to traffic surveillance. Researchers are frequently choosing quadrotors for their research because a quadrotor can accurately and efficiently perform tasks that would be of high risk for a human pilot to perform. The quadrotor has an advantage of maneuverability due to its inherent dynamic nature. It is an under-actuated system with four inputs (roll, pitch, yaw and throttle) and six outputs. The parameters that determine the characteristics of a flying machine are the flying principle and propulsion mode[Gupte, 2012].

With the increase in attention UAVs and quadrotors have received in the last decade, the algorithms laid out to control them have also increased substantially in number and complexity. Various control structures ranging from basic PID controllers [Erginer, 2007]to more complex systems using backstepping or neural networks have proved to be efficient. There has been considerable innovation in the sensors that are used to control the quadrotor as well. Modern MEMS technology makes it easier to add more sensors on a small quadrotor as the space and weight constraints can be stringent. Sensors including basic IMUs, GPS modules and cameras have all been used in quadrotors[Madani, 2006]. The general method to design a control system is to calculate the dynamic model of the system. The dynamic model of a system is a mathematical equation that comprises of all the forces that can act on the system at a given time. Many researchers have also tried comparing different control techniques and in most cases a quadrotor proved to be a dynamic vehicle with major challenges because of its under-actuated nature. Algorithms like PID, backstepping and feedback linearization have mainly been applied and proved to work well with a quadrotor. A typical quadrotor will have parts like ESCs, four rotors, an IMU and a microcontroller running a control algorithm. Parts like vision based system and GPS are optional. As explained in Section I, the quadrotor is controlled using the values of its throttle, roll, pitch and yaw. The control algorithm reads the IMU sensor values and modifies these values in the opposite direction to stabilize the system. There have also been control methodologies which have found their motivation in nature[Tsourdos, 2005]. Quadrotor control is based upon interactions of animals and insects in nature. The control algorithm is developed in a way that it can be used in a multi-agent environment, where each quadrotor can predict the location of the other. Control systems are also designed specifically for multi-agent systems [Tsourdos, 2005] where the quadrotors work along with each other to perform a specific task. Another major difficulty that is faced in control of a quadrotor is forces of nature. Factors like wind and terrain play a very important factor when flying in an open space. Control systems [Mokhtari, 2004]for these conditions are also performed where wind parameters are estimated and the different degrees of freedom are control based on these parameters

In the article, the object of research is a UAV (quadrocopter), the capabilities of which allow it to be used in any natural and technical conditions without preliminary preparation of the take-off and landing zone. The purpose of this work is to study the UAV flight altitude stabilization system using the example of a quadrocopter. The main tasks of the study: research of the AR.Drone2.0 
model; analysis of the results obtained.

\section{MATERIALS AND METHODS}

UAV is a type of aircraft that is piloted remotely, that is, without the presence of a person on board. The first radio-controlled ship was designed by Nikola Tesla in 1898. The breakthrough occurred only in 1910, when an American military engineer proposed the option of using the aircraft remotely, that is, without the participation of a pilot, but, unfortunately, did not receive development due to one-time use of the apparatus. Since 1933, the active development of UAVs in the military industry begins. Over the next 2-3 decades, several models were developed and successfully applied. Recently, there has been a boost in the development of UAVs for mass use. The most famous developments of the time, the DH.82B Queen Bee, were used in Great Britain. According to media reports in 2012, the armed forces included more than 7494 guided aircraft, while the number of UAVs exceeded 11 thousand units. Today, UAVs have gained massive popularity, which served as a new round in development. More and more new models are emerging aimed at the use of civil services. In this regard, the models are becoming more affordable, easy to operate, and their dimensions are becoming smaller, which in turn affects the technical aspect of development.

Quadrocopter - UAV with four rotors. Changing the speed of screw rotation allows you to control the movement of the apparatus.

A multicopter is an aircraft with three or more rotors located in one plane and creating the lift required for flight. There are several types of multicopter: tricopter (three carrier motors), quadrocopter (four carrier motors), hexacopter (six carrier motors), etc.

The earliest experimental takeoff attempts with a rotorcraft drone were mostly accomplished using a multitron. Around 1907 Jacques and Louis Breguet, brothers in France, built and tested Gyroplane No. 1, a quadcopter. They managed to take off, although the model proved to be very unstable and impractical. In 1924, French engineer Etienne Ohmichen flew $360 \mathrm{~m}$ in his quadcopter, setting a world record. In the same year, he flew a circle with the diameter of $1 \mathrm{~km}$. Around the same time, George de Botezat built and tested his quadcopter for the US Army, completing a series of test flights before the program was closed. It can be concluded that the quadcopter is the most versatile UAV model.

"Parrot" company was one of the first to release a quadrocopter for the mass consumer - AR.Drone 2.0 (Figure 1) (https://mobile-review.com). Four electric motors have the power of $14.5 \mathrm{~W}$ each, providing $28500 \mathrm{rpm}$, maximum flight speed $\sim 18 \mathrm{~km} / \mathrm{h}$. The body is equipped with two HD video cameras.

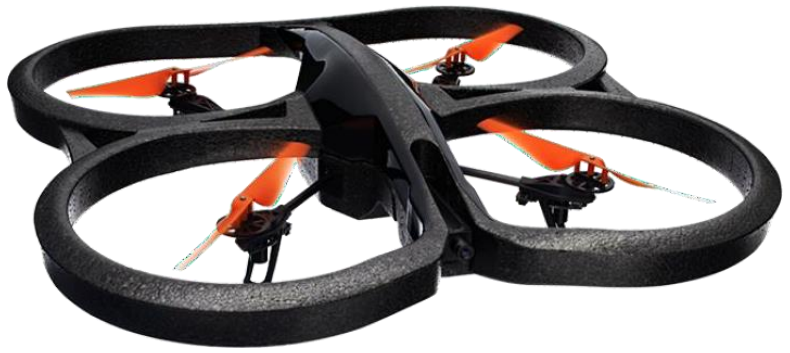

Figure 1: Quadcopter AR.Drone 2.0

Control takes place over a Wi-Fi network. AR Drone 2.0 is one of the best Wi-Fi enabled quadcopters on the market. The superiority of this model lies in the fact that it is universal. For orientation in space, the following sensors are installed on the copter: a three-axis gyroscope, a three-axis accelerometer, a three-axis magnetometer (magnetic compass), a pressure sensor and 
an ultrasonic altimeter. It should be noted that the microphone is missing, because it will be useless at this noise level.

The emergency power cut-off button is designed to instantly turn off the power of the screws, which can lead to a fall. In any other case, the quadrocopter sits down by itself or waits for the landing command. Battery resources (voltage $11.1 \mathrm{~V}, 1000 \mathrm{mAh}$ ), provides up to twelve minutes of continuous flight, i.e. the quadcopter, in principle, is not designed for long-term continuous operation.

The principle of a quadcopter flight. The quadcopter is designed in the form of two mutually perpendicular beams with the sides of equal length. A propeller with a drive is attached to the end of each beam. The propellers are fixed by the locking axes. A lift is generated with the simultaneous operation of propellers; it acts perpendicular to the quadcopter itself. The lifting force depends entirely on the speed at which the propellers rotate.

The quadcopter has four main axes along which control is carried out: flight altitude, pitch, roll about the longitudinal axis, yaw along the course.

Angular degrees of the quadcopter motion:

- Heading (turn parallel to the takeoff surface): controlled by increasing the rotational speed of the rotors turning in the direction of the turn and decreasing the speed of the counter-rotating rotors. There is no change in altitude when turning on the heading since power is supplied to the twin motors symmetrically. The main problem with yaw control is the choice of motors according to which the power is increased or decreased.

- Roll (tilts to the right and left): control occurs due to a symmetrical change of the rotor power. Power increases on one rotor, and decreases on the other.

- Pitch (tilts up and down): controlled by analogy with roll, but with a different pair of motors.

\section{Flight stabilization principle}

A quadrocopter is an aircraft, in accordance with this, the following aviation names are used for the tilt angles, also shown on Figure 2 (Bouabdallah, 2007; Geng \& Chulin, 2015): pitch in the plane on the $\mathrm{X}$ coordinate axis, roll on the $\mathrm{Y}$ axis and, accordingly, the course on $\mathrm{Z}$ axis.

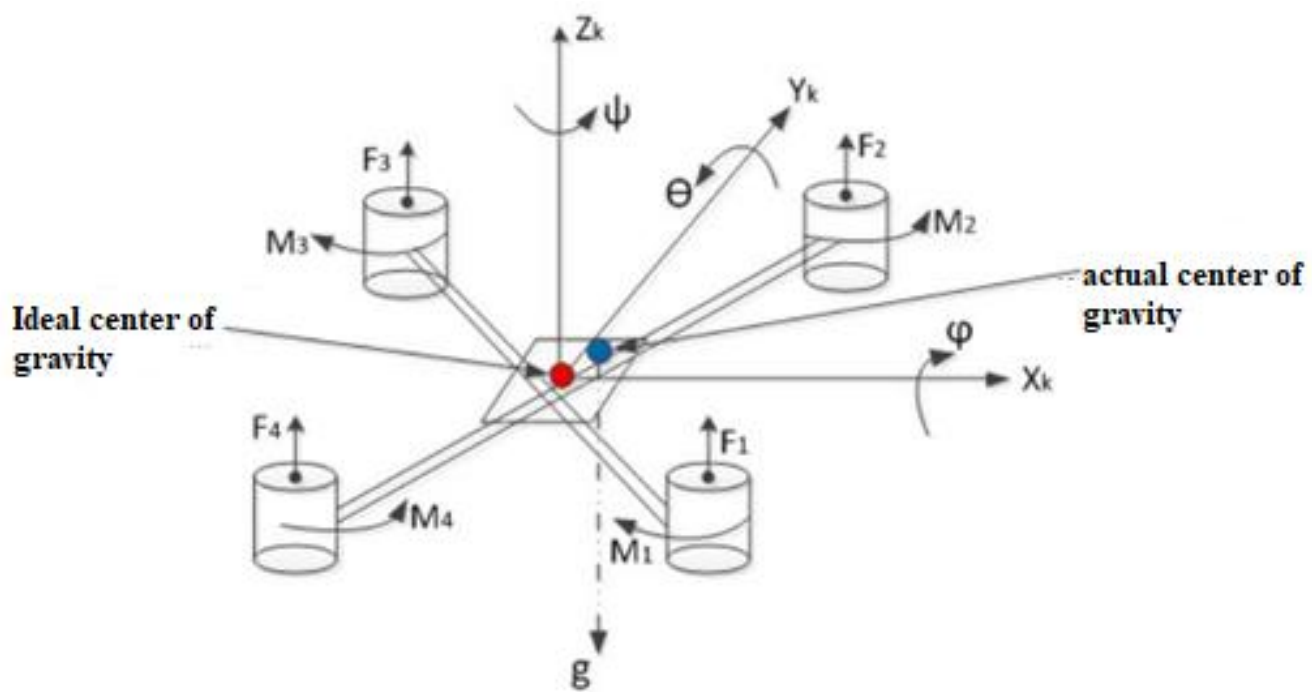

Figure 2: Correspondence of inclination angles and planes

Integrating the data obtained from the gyroscope, it is possible to determine the angle, a frequently used method is the method of rectangles (Samarsky \& Gulin, 1989): 


$$
\alpha=\alpha_{0}+\omega t
$$

where $\alpha$-the current value of the angle, $\alpha_{0}$ - the previous value of the angle, $\omega$ - the current value of the angular velocity, $t$ - the time between measurements.

A significant disadvantage is that the error resulting from the measurement error of the gyroscope is also summed up. With time increase, the error will increase significantly, which will lead to a strong discrepancy with the real values. Accordingly, it is necessary to periodically correct the calculated angles so that the values do not "float away" from the real indicators. An accelerometer is used for such corrections.

The system is at rest or moving at a constant speed, i.e. since there is no natural acceleration, the tilt angle can be calculated using the following expressions (triaxial case).

$$
\begin{aligned}
& \varphi=\arctan \left(\frac{A_{x}}{\sqrt{A_{y}^{2}+A_{z}^{2}}}\right), \\
& \theta=\arctan \left(\frac{A_{y}}{\sqrt{A_{x}^{2}+A_{z}^{2}}}\right), \\
& \psi=\arctan \left(\frac{A_{z}}{\sqrt{A_{y}^{2}+A_{x}^{2}}}\right) .
\end{aligned}
$$

where $A_{x}, A_{y}, A_{z}$ - the projections of the gravitational acceleration vector onto the projections of the gravitational acceleration vector on the corresponding $\mathrm{X}, \mathrm{Y}, \mathrm{Z}$ axes.

Due to the accelerometer, it is possible to correct only the roll and pitch angle. The heading angle cannot be corrected because the axis of rotation coincides with the rotation axis of the gravitational acceleration projection sum g. This problem is solved using a magnetometer. An equally important link in flight stabilization is an altimeter, which maintains a given altitude, as well as a safe landing when the emergency mode is turned on.

The analysis of world publications has shown that there are many articles and works that consider possible ways to stabilize a quadrocopter, including the development of a dynamic model, control algorithms and experimental studies of real-life model movement. The control method is most often based on PD or PID controllers (Khalil, 2009), as well as linear-quadratic controllers (LQC) (Shepherd, 2010), fuzzy controllers (Hong, 2005) and the recursive stabilization method (in English Backstepping) (Buchholz \& Gretarsson, 2009). In the course of the study of the available materials of the Russian-language literature, almost no scientific works and articles were found that dealt with the topic of stabilization of quadrocopters. The most complete and informative are the articles by S.A. Belokon and other researchers from Novosibirsk (Belokon, 2012).

The scientists at the MTI Aerospace Laboratory have developed a control method that is unique: it uses variable pitch and follower propellers instead of a standard propeller. Thanks to this, you can change the lifting force by adjusting the speed of screw rotation. But the angle of attack can also be changed, which makes it possible to increase manoeuvrability, as well as turn the platform of the device and fly upside down. The developers have modeled and tried to test this design and verification method in real experiments. 
Researchers at Stanford University describe a control method that also uses a PID controller to control the flight angle and controllers. The work is characterized by a deep study of the quadrocopter model dynamic motion in relation to the aerodynamics of the fuselage (Xian et al., 2015).

The scientific community of "KumarLab" laboratory workers, the University of Pennsylvania, under the supervision of Professor Kumar. Thanks to the motors based on the PD controller, as well as the system that automatically creates the best flight route, the quadcopter presented by this team is much faster and more maneuverable than the standard model. It is stated that the micro quadrocopter (with the dimensions from 0.1 to $0.5 \mathrm{~m}$ and the weight from 0.1 to $0.5 \mathrm{~kg}$ ) is much more maneuverable. In this REGARD, special attention must be paid to the model weight and size. The scientists from the University of Pennsylvania have successfully solved the problem avoiding collision with dynamic obstacles. The solution to the problem is that the control system constantly needs the exact coordinates of the aircraft. To remedy this deficiency, the team led by Kumar developed a prototype equipped with a wide range of sensors, including a GPS sensor, an on-board camera and a laser scanner. The model can be launched both outdoors and indoors. A 3D model/map of the territory is created during the flight (Bouabdalla \& Siegward, 2005).

\section{Mathematical model of a quadrocopter}

When simulating the flight of a quadcopter, it is necessary to take into account its capabilities, namely the flight time and range (Guryanov, 2014; Mesyats, 2015). We take the earth as an absolutely inertial coordinate system, the wind is conventionally taken as an external influence. The relative position of the coordinate systems $(O X Y Z)$ and $\left(O_{0} X_{g} Y_{g} Z_{g}\right)$, the normal earth at rest and the forces and moments acting on the quadcopter are shown on Figure 3.

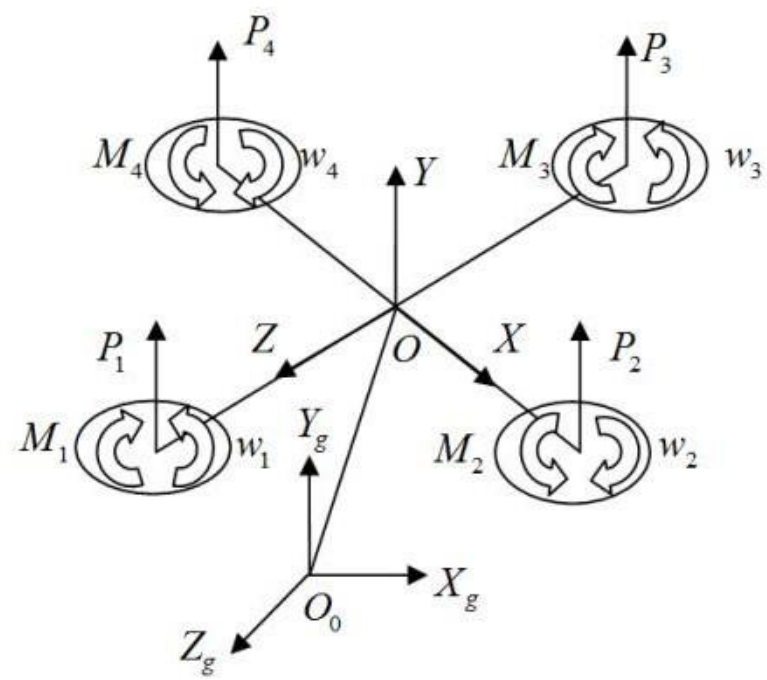

Figure 3: Mutual position of coordinate systems

Figure 3: $P_{i}(i=1,2,3,4)$ - the thrust force of the corresponding screw, $M_{i}(i=1,2,3,4)-$ the moment from the resistance of the corresponding screw, $\quad w_{i}(i=1,2,3,4)$-the rotation speed. The thrust forces are connected and represented by one coordinate system (Golubev, 2000) 


$$
\left\{\begin{array}{l}
P_{b}=\left[\mathrm{P}_{\mathrm{x}}, \mathrm{P}_{\mathrm{y}}, \mathrm{P}_{\mathrm{z}}\right]^{\mathrm{T}}=[0, \mathrm{P}, 0]^{\mathrm{T}} \\
P=P_{1}+P_{2}+P_{3}+P_{4}=\sum_{i=1}^{4} c_{p i} w_{i}^{2} \\
c_{p i}=\frac{1}{2} \rho c_{a} S_{i} r_{i}^{2}
\end{array}\right.
$$

where $P$ - the total thrust,

$c_{p i}$ - traction coefficient,

$\rho$ - air density,

$c_{a}$ - liftig force ratio,

$S_{i}$ - the area of the surface swept by the blades of the i-th propeller,

$r_{i}-$ the $\mathrm{i}$-th screw radius.

In standard coordinate system:

$$
P_{g}=\left[\mathrm{P}_{\mathrm{Xg}}, \mathrm{P}_{\mathrm{Yg}}, \mathrm{P}_{\mathrm{Zg}}\right]^{\mathrm{T}}=\mathrm{RP}_{\mathrm{b}},
$$

where

$$
R=\left[\begin{array}{ccc}
\cos \theta \cos \psi & -\cos \gamma \cos \psi \sin \theta+\sin \gamma \sin \psi & \sin \gamma \cos \psi \sin \theta+\cos \gamma \sin \psi \\
\sin \theta & \cos \gamma \cos \theta & -\sin \gamma \cos \theta \\
-\cos \theta \sin \psi & \cos \gamma \sin \psi \sin \theta+\sin \gamma \cos \psi & -\sin \gamma \sin \psi \sin \theta+\cos \gamma \cos \psi
\end{array}\right]-\text { transition }
$$
matrix, $\psi, \theta, \gamma$-heading, pitch and roll angles, respectively.

Let us put down the expression to describe the force of gravity and the force of resistance in the following form:

$$
\left\{\begin{array}{l}
f=\left[-f_{\mathrm{x}},-f_{\mathrm{y}},-f_{\mathrm{z}}\right]^{\mathrm{T}} ; \\
G=[0,-\mathrm{mg}, 0]^{\mathrm{T}} .
\end{array}\right.
$$

The system of equations for the dynamics of mass center motion in the normal terrestrial coordinate system will look like this.

$$
\left\{\begin{array}{l}
\ddot{x}=\frac{\left[P(-\cos \gamma \cos \psi \sin \theta+\sin \gamma \cos \psi)-f_{\mathrm{x}}\right]}{m} ; \\
\ddot{y}=\frac{\left[P(\cos \gamma \cos \theta)-f_{\mathrm{y}}-m g\right]}{m} ; \\
\ddot{z}=\frac{\left[P \cos \gamma \sin \psi \sin \theta+\sin \gamma \cos \psi-f_{\mathrm{z}}\right]}{m} .
\end{array}\right.
$$

Let us describe a situation in which the mass center is located at the origin of the bound system coordinates. Then the equation for the dynamics of angular motion in a coupled system will have the following form. 


$$
\begin{aligned}
& \left\{\begin{array}{l}
\dot{w}_{x}=\frac{I y-I z}{I x} w_{y} w_{z}+\frac{M_{R x}}{I x} \\
\dot{w}_{y}=\frac{I z-I x}{I y} w_{x} w_{z}+\frac{M_{R y}}{I y} \\
\dot{w}_{z}=\frac{I x-I y}{I z} w_{x} w_{y}+\frac{M_{R z}}{I z}
\end{array}\right. \\
& \left\{\begin{array}{l}
M_{R x}=M_{q x}+M_{m x}+M_{p x} \\
M_{R y}=M_{q y} \\
M_{R z}=M_{q z}+M_{m z}+M_{p z}
\end{array}\right.
\end{aligned}
$$

where $w_{x}, w_{y}, w_{z}$ - the projections of the angular velocity vector of the vehicle onto the associated coordinate system; $M_{R x}, M_{R y}, M_{R z}$ - the projections of the resultant moment; $M_{q x}, M_{q y}, M_{q z}-$ the moments created by the screws; $M_{m x}, M_{m z}, M_{p x}, M_{p z}$ - gyroscopic moments of motors and propellers.

Omitting the fact of screw inertia presence when the angular velocities of their rotation change, these moments can be represented as the following system of equations.

$$
\begin{aligned}
& \left\{\begin{array}{l}
M_{q x}=\left(P_{3}-P_{1}\right) l \\
M_{q y}=M_{2}+M_{4}-M_{1}-M_{3} ; \\
M_{q z}=\left(P_{2}-P_{4}\right) l ; \\
M_{i}=m_{P i} w_{i}^{2} \\
m_{P i}=\frac{1}{2} \rho c_{a} S_{i} r_{i}^{2} .
\end{array}\right. \\
& \left\{\begin{array}{l}
M_{m x}=\mathrm{I}_{\mathrm{m}} w_{z}\left(w_{1}+w_{3}-w_{2}-w_{4}\right) ; \\
M_{m z}=\mathrm{I}_{\mathrm{m}} w_{x}\left(w_{2}+w_{4}-w_{1}-w_{3}\right) ; \\
M_{p x}=\mathrm{I}_{p} w_{z}\left(w_{1}+w_{3}-w_{2}-w_{4}\right) ; \\
M_{p z}=\mathrm{I}_{p} w_{z}\left(w_{2}+w_{4}-w_{1}-w_{3}\right) .
\end{array}\right.
\end{aligned}
$$

where $l-$ the distance from the mass center to the screw axis; $\mathrm{I}_{\mathrm{m}}, \mathrm{I}_{p}-$ the moments of the rotor and screw inertia; $m_{P i}-$ the torque coefficient.

The creation and study of a mathematical model will be carried out in the Matlab Simulink software environment used for a convenient modeling process of motion equation integration dynamics. The model in Matlab Simulink contains four blocks: input voltage; voltage limiters; propeller engine model; quadcopter model.

Control voltage for motors, the input signal of this work, the output signal is described by the 
coordinates $(\mathrm{x}, \mathrm{y}, \mathrm{z})$ and the angles $(\gamma, \psi, \theta)$. Control voltages on motors with leading distributors of signal limiters and voltages. For the selected quadcopter linear speed versus voltage control $w_{i}=100,8 u_{i}$ within the operating speed range, the input voltage is limited to the values from 0 to $11.1 \mathrm{~V}$. The above thrust forces are perpendicular in any situation to the quadcopter platform plane. The value of the forces, as well as the moments $M_{m}, M_{P}, M_{q}$, acting on the quadcopter, are taken from the block created in the Matlab program.

\section{Stabilization algorithm}

Flight stabilization is one of the main components of a quadcopter. The system is presented as a sequence of flight tasks to the next specified route point or movement along specified segments of typical trajectories. In this case, the automatic control system can be built as a feedback system tracking a given route. The system that independently tracks a given path.

Figure 4 illustrates the structure of the control system (Geng \& Chulin, 2015):
1 - Specified trajectory;
2 - Corrective devices (CD) of the trajectory control subsystem;
3 - Coordinate converter;
4 - Regulator of the orientation and stabilization subsystem;
5 - Signal distributor;
6 - Voltage limiter;
7 - Propeller group model;
8 - Quadcopter model.

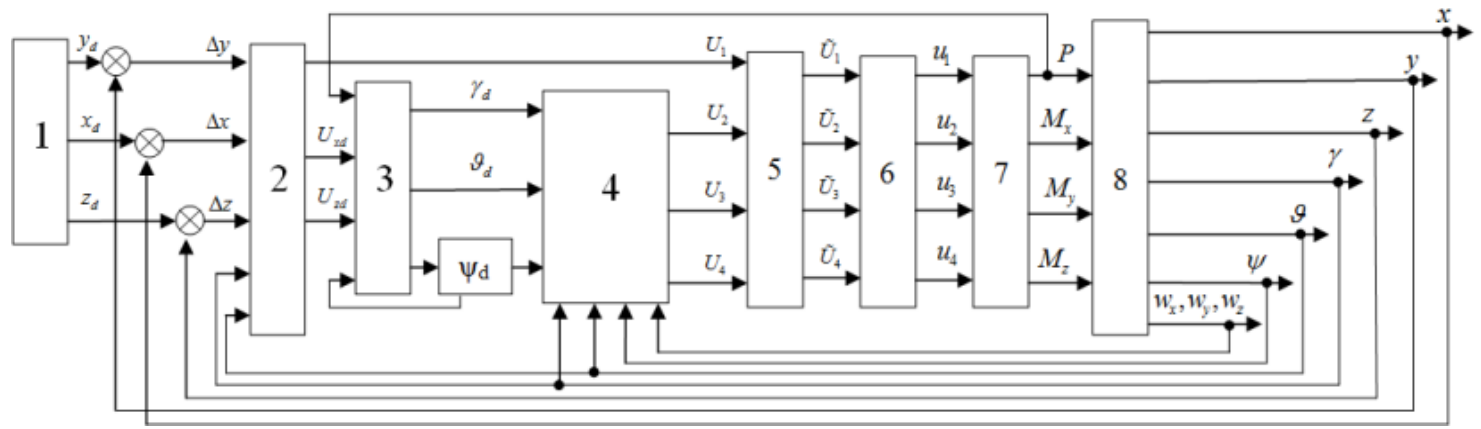

Figure 4: Control system structure

To calculate control algorithms by stabilizing the operation of subsystems, one of the possible methods should be used. It is better to choose the methods that are presented as the simplest. For each subsystem, a separate calculation of corrective elements, such as PID, is carried out using the method called "backstepping" (Bouabdalla \& Siegward, 2005; Chebykin, 2013). Upon completion of the comparison results, an optimal controller for each of the subsystems will be selected.

\section{Angular position stabilization algorithms}

The signals are input subsystems: stabilization and orientation of angular positions. In turn, the output subsystems are the parameters of the angular motion of an object.

The control algorithm is a subsystem that generates the control signals $U_{2}, U_{3}, U_{4}$, which also provide the generation of the moments $M_{R x}, M_{R y}, M_{R z}$ (the projections of the resultant moment), provided that the total thrust of the propellers is sufficient for vertical movement (Kovalev et al., 
2015). Neglecting the dynamics and limitations of the engines, gyroscopic moments and aerodynamic moments of the non-carrying part of the apparatus will lead to the fact that the signals $U_{2}, U_{3}, U_{4}$ should be correspondingly equal, up to tenths, to the moments $M_{R x}, M_{R y}, M_{R z}$. Based on the above, the equations (5) and (6) are suitable for the stabilization algorithm of the object model at $M_{R x}=U_{2}, M_{R y}=U_{3}, M_{R z}=U_{4}$.

For the angular motion of an aircraft, such a case is possible at small pitch and roll angles, when the derivatives of the angles $\gamma, \psi, \theta$ can be considered equal to the corresponding angular velocities. Then the equations of angular motion (7.8) can be approximately represented in the form of three subsystems:

$$
\left\{\begin{array}{l}
S_{1}=\left\{\begin{array}{l}
\dot{\gamma}=w_{x} ; \\
\dot{w}_{x}=\frac{I y-I z}{I x} w_{y} w_{z}+\frac{M_{R x}}{I x}
\end{array}\right. \\
S_{2}=\left\{\begin{array}{l}
\dot{\psi}=w_{y} ; \\
\dot{w}_{y}=\frac{I z-I x}{I y} w_{x} w_{z}+\frac{M_{R y}}{I y} ;
\end{array}\right. \\
S_{3}=\left\{\begin{array}{l}
\dot{\theta}^{\prime}=w_{z} \\
\dot{w}_{z}=\frac{I x-I y}{I z} w_{x} w_{y}+\frac{M_{R z}}{I z}
\end{array}\right.
\end{array}\right.
$$

\section{«Backstepping» method}

Backstepping - a recursive process in which the problems of the Lyapunov function and the corresponding control laws appear (Bouabdalla \& Siegward, 2005; Chebykin, 2013). In accordance with this method, the problem of the law for the entire system control is divided into a sequence of corresponding subtasks for the subsystems of smaller size (or scalar systems). Since a programmer has unlimited freedom in the analysis of scalar and small-order systems, this method helps to solve the problems of stabilization and tracking relatively easily using robust control under less restrictive conditions, in contrast to other methods (Khalil, 2009).

\section{Trajectory control algorithm}

If we follow the equation of dynamics for the projection of the horizontal force, excluding the resistance of the non-bearing part of the apparatus, it will take the following form.

$$
\begin{gathered}
U_{x}=P(-\cos \gamma \cos \psi \sin \theta+\sin \gamma \sin \psi) \\
U_{z}=P(\cos \gamma \sin \psi \sin \theta+\sin \gamma \cos \psi)
\end{gathered}
$$

From these expressions, it is possible to determine the roll and pitch angles at which the required actions are created with a known total thrust $P$.

$$
\begin{array}{r}
\gamma_{d}=\arcsin \frac{U_{z d} \cos \psi_{d}+U_{x d} \sin \psi_{d}}{P} \\
\theta_{d}=\arccos \frac{U_{z d} \sin \psi_{d}+U_{x d} \cos \psi_{d}}{P}
\end{array}
$$

The control actions $U_{x d}$ and $U_{z d}$, as well as $U_{y d}=U_{1}$ for the height control channel, can be obtained by considering the trajectory control subsystem as a control system that tracks the 
required coordinates of the mass center, in particular, as the output signals of the PID controller according to the deviations of the mass center coordinates from the required ones.

\section{RESULTS AND DISCUSSION}

The performance of the algorithm was tested by modeling the responses to step effects and flight along specified trajectories. The simulation scheme in the Matlab Simulink environment is shown on Figure 5, and the model responses to a step action are shown on Figures 6 and 7.

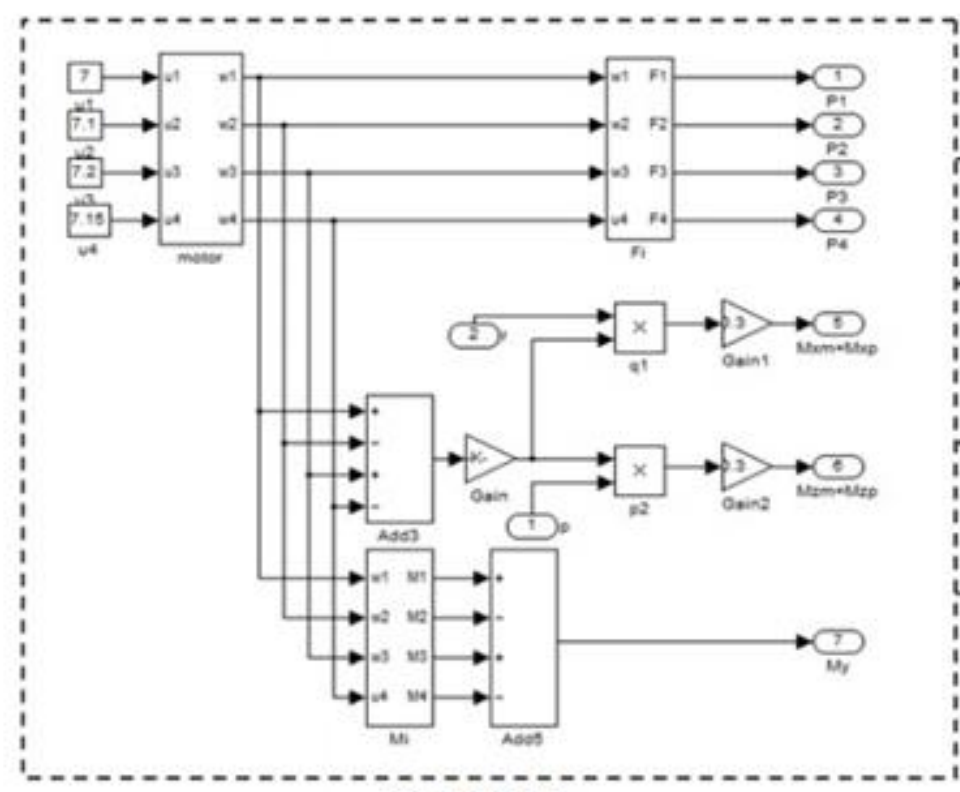

Unit Matlab

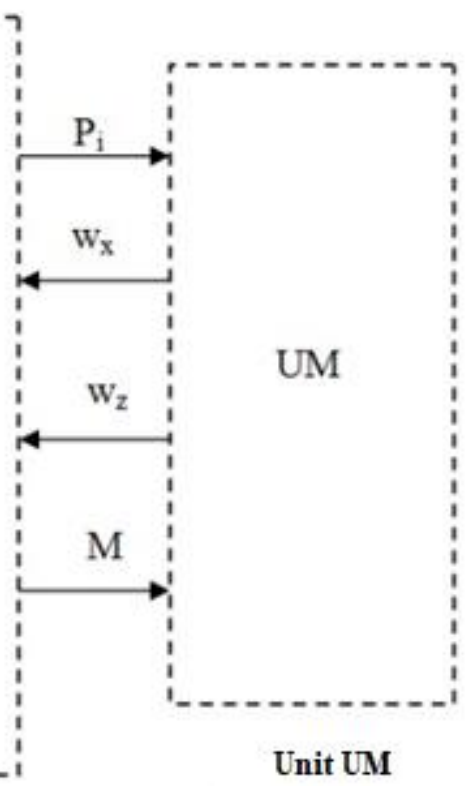

Figure 5: Modeling scheme
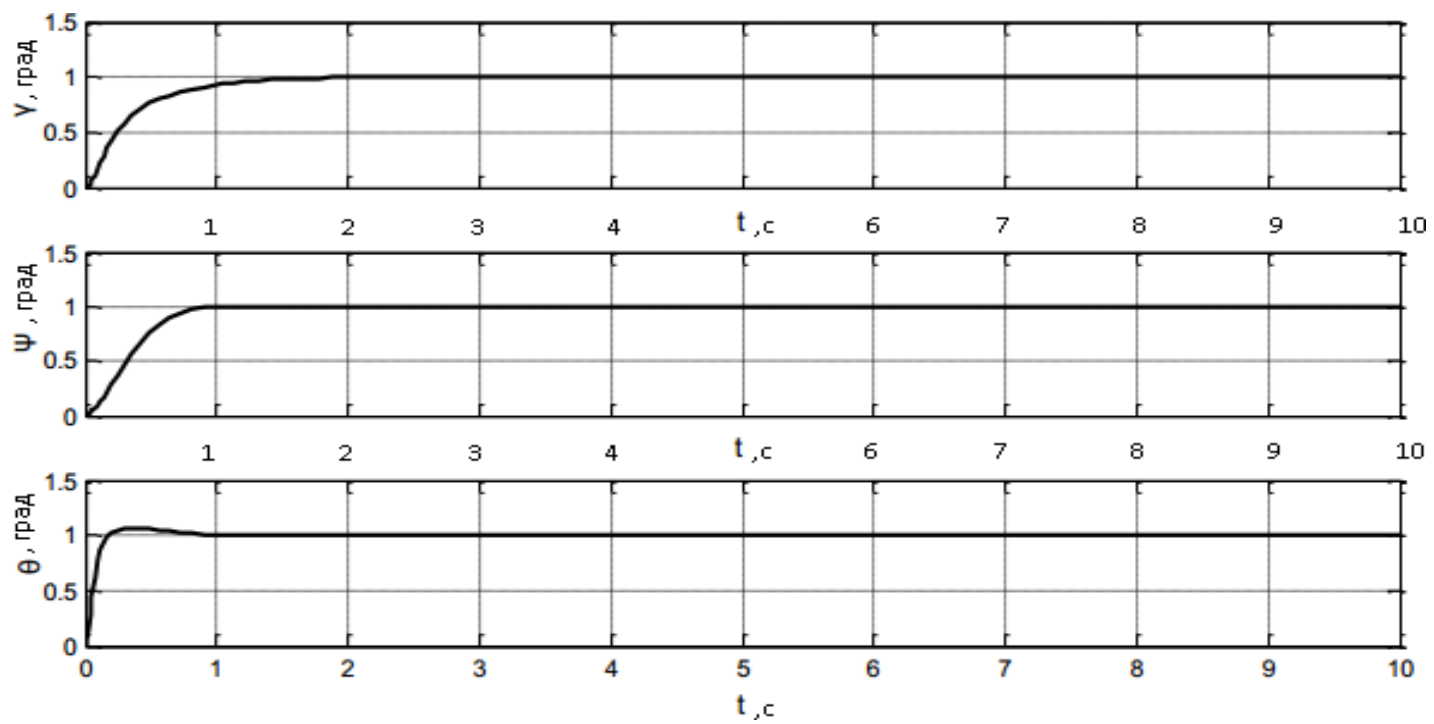

Figure 6: Reactions to stepwise action $(\gamma, \psi, \theta)$ 

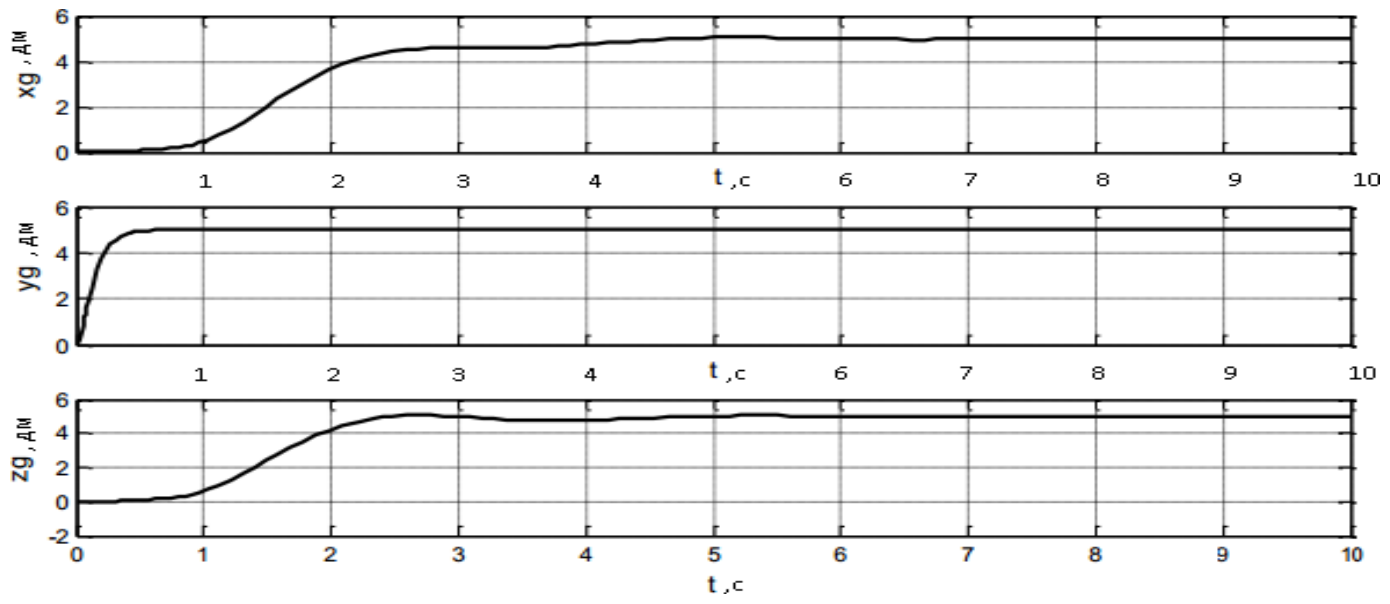

Figure 7: Reactions to stepwise action $\left(x_{g}, y_{g}, z_{g}\right)$

\section{CONCLUSION}

The article proposes a quadrocopter mathematical model. A simple control algorithm is presented that allows you to stabilize the height and angular position. The results obtained during the study show the algorithm efficiency and the possibility of its practical implementation.

The developed mathematical model can be used instead of a real quadcopter, this will significantly reduce the time during research, as well as avoid the quadrocopter damage, reducing the number of launches.

\section{REFERENCES}

Belokon, S.A. (2012). Quadrocopter flight parameter control moving along a given trajectory. Avtometriya, 5, 32-41.

Bouabdalla, S., \& Siegward, R. (2005). Backstepping and sliding-mode techniques applied to an indoor micro quadrotor. IEEE International Conference on Robotics and Automation, 2259-2264.

Bouabdallah, S. (2007). Design and Control of quadrotors with application to autonomous flying. Lausanne, EPFL, $129 \mathrm{p}$.

Buchholz, T.T., \& Gretarsson, D. (2009). Construction of a Four-Rotor Helicopter Control System: S.M. Thesis. Technical University of Denmark, 158 p.

Chebykin, D.V. (2013). Backstepping - the control synthesis method for nonlinear objects, Ural Federal University named after the first President of Russia B.N. Yeltsin -. 254 p.

Geng, Ke. Ke., \& Chulin, N.A. (2015). Multi-mode stabilization controller for automatic control of a quadrocopter trajectory motion. International Journal of Experimental Education, 12(3), 428-435.

Golubev, Yu.F. (2000). Fundamentals of theoretical mechanics, 2nd ed., M.: Publishing house of Moscow State University, $720 \mathrm{p}$.

Guryanov, A.E. (2014). Simulation of a quadrocopter control. Engineering Bulletin, 8, 522-534.

Hong, S.K. (2005). Fuzzy logic based closed-loop strap down attitude system for unmanned aerial vehicle (UAV), Elsevier B.V, 110-126. 
Khalil, H.K. (2009). Non-linear systems. - M. - Izhevsk: Research Center "Regular and Chaotic Dynamics", Institute of Computer Research, - 832 p.

Kovalev, E.S., Bezzubtsev, A.Yu., \& Smirnov. A.V. (2015). Development of algorithms. Science-intensive information technologies., 6-15.

Mesyats, A.I. (2015). Control problems for the systems with ellipsoidal dynamics: abstract from the dis. by $\mathrm{PhD}$ in physics and mathematics. Moscow. 26 p.

Mobile-review.com. Parrot AR. Drone 2 review. Power Edition: [Electronic resource] // mobile-review.com. URL: https://mobile-review.com/articles/2015/parrot-ardrone2-pe.shtml. (Reference date: 18.06.2020).

Samarsky, A.A., \& Gulin, A.V. (1989). Numerical methods: Textbook for universities. - M.: Science. Ch. ed. of phys-math. lit., - $432 \mathrm{p}$.

Shepherd, J. (2010). Robust Neuro-Control for a Micro Quadrotor / Shepherd J., Tumer K.// GECCO' 10. Portland, Oregon, USA. 9 p.

Xian, B., Diao, C., Zhao, B., \& Zhang, Y. (2015). Nonlinear robust output feedback tracking control of a quadrotor UAV using quaternion representation. Nonlinear Dynamics, 79(4), 2735-2752. 